\title{
Crossing Rivers Problem Solution with Breadth-First Search Approach
}

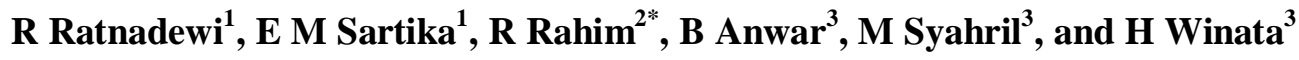 \\ ${ }^{1}$ Electrical Engineering, Maranatha Christian University, Jalan Prof. Drg. Surya \\ Sumantri No.65, Bandung 40164, Indonesia \\ ${ }^{2}$ Department of Health Information, Akademi Perekam Medik dan Infokes Imelda, \\ Jl. Bilal Ujung Medan 20116, Indonesia \\ ${ }^{3}$ Department System Computer and Information System, STMIK Triguna Dharma, \\ Jl. Jenderal Abdul Haris Nasution No.73, Medan 20219, Indonesia \\ *usurobbi85@zoho.com
}

\begin{abstract}
Crossing river problem is one of the problems state and space that represents a state by using a rule to define the problem, to find solutions to state and space problem can use breadth first search method by conducting a search based on rule and condition given, it is expected with this research crossing river problem could be solved properly and optimally and also this research can be the basis of solving other similar problems by using breadth first search method, to model the situation and the problem space using Breadth First Search made an application with a programming language.
\end{abstract}

\section{Introduction}

A state is a representation of a state at a time or decryption of system configuration [1] [2]. State and space are all possible states and usually described as networks with vertices being the state and edge representing possible changes [1]. The representation of state and space allows the formal definition of a problem as a matter of changing status by using a set of operators (rules) and also defining a problem as a search i.e. searching the path in state and space from the initial state to the goal state [1] [2].

Problem state and space [1] is a common problem in the field of Artificial Intelligence, Searching Process and also Machine Learning, some examples of state and space problems such as 8-Puzzle, Cannibals and Missionaries Problem and also Water Jug Problem, and on the results of this study conducted experiments with create a problem called Crossing River Problem.

The illustration of this Crossing River Problem is as follows, a family will cross to the other side of the river along with police and criminals, as for the variables of crossing rivers problem consisting of father, mother, son 1, son 2, daughter 1, daughter 2, criminals and police.

This problem will be modified so that it can be used to solve all problems that are almost similar to this issue. This issue could be resolved by using several stages, the first stage starts from the identification of the state space, by declaring this problem by determining the variables contained in this problem. Then, specify the rules included in the problem of initial conditions and conditions of purpose, crossing rivers problem solved using the help of tracking tree. Possible conditions are described in the tree structure starting with the first state as the root of the tree. The process is continued by describing 
the next state that could be created from that state. The process continues until a goal state is obtained or no new conditions can be developed.

Breadth-first search (BFS) search method is used to perform checking procedures whether every action carried out on any circumstances violates pre-made rules [3]. This procedure ensures that new conditions to be established do not violate existing rules so that the desired goal state can be obtained, to facilitate the experiment of using Breadth-First Search algorithm, an application was created to model the BFS simulation process to solve the problem created.

\section{State and Space Problem}

Systems that use artificial intelligence will try to provide output in the form of a solution of a problem based on a collection of existing knowledge [1] [4] [5]

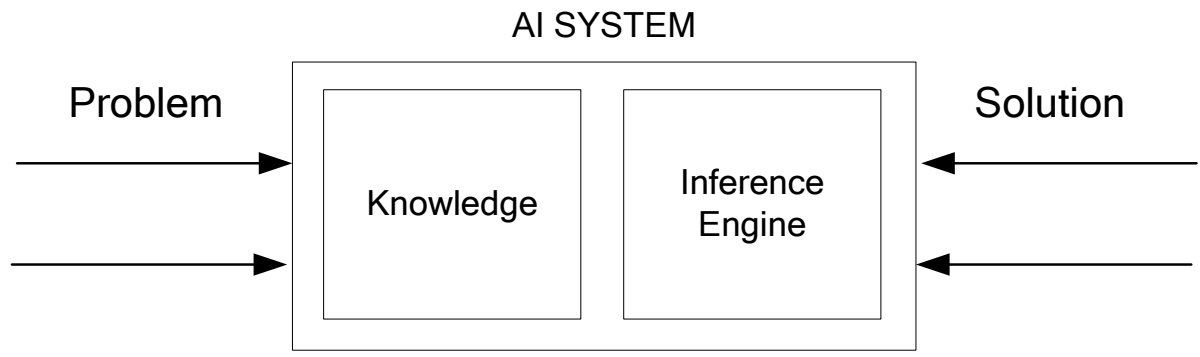

Figure 1. Systems that use AI

Input given to systems using artificial intelligence is a problem, on the system must be equipped with a set of existing knowledge in the knowledge base. The system must have an inference engine to be able to draw inferences based on fact or knowledge. The given output is a problem solution as a result of inference [1] [4] [6]. In general, to build a system capable of solving problems, four things need to be considered:

a. Define the problem appropriately. This definition includes precise specifications of the initial state and the expected solution.

b. Analyze the problem and look for some appropriate problem-solving techniques.

c. Represents the necessary knowledge to solve the problem.

d. Choosing the best problem-solving technique

\section{Breadth-First Search}

In the Breadth-First Search method, all nodes at level $\mathrm{n}$ will be visited first before visiting nodes at level $n+1$ [3] [6] [7]. The search starts from the root node and continues to the 1st level from left to right, then moves to the next step. Moreover, so on until the discovery of a solution [5] [6] [7].

The advantages of this breadth-first search method are [6]:

a. Will not get stuck.

b. If there is one solution, then a breadth-first search will find it. Also, if there is more than one solution, then a minimum solution will be found.

The weakness of this method [6]:

a. Requires enough memory, because it stores all the nodes in one tree.

b. It takes a long time because it will test $n$ level to get the solution at level $(n+1)$.

\section{Result and Discussion}

Breadth First Search algorithm completion on crossing rivers problem. The illustration of this problem is as follows, a family will cross to the other side of the river along with police and criminals, as for the variables of crossing rivers problem consisting of father, mother, son 1, son 2, daughter 1, daughter 2, criminals and police, Table of variables and rules can be seen in table 1 and table 2 . 
Table 1. Variable Crossing Rivers Problem

\begin{tabular}{|c|c|c|}
\hline Variable ID & Variable Name & Could Raft Driver \\
\hline AY & Father & True \\
\hline IB & Mother & True \\
\hline AP1 & Daughter 1 & False \\
\hline AL1 & Son 1 & False \\
\hline AL2 & Son 2 & False \\
\hline AP2 & Daughter 2 & False \\
\hline PL & Police & True \\
\hline PJH & Criminal & False \\
\hline
\end{tabular}

Table 2. Crossing Rivers Problem Rules

\begin{tabular}{|c|l|}
\hline No & \multicolumn{1}{|c|}{ Rule } \\
\hline 1 & Father will beat Girl 1 if Mother is not in place \\
\hline 2 & Father will beat Girl 2 if Mother is not there. \\
\hline 3 & Mother will hit Son 1 if Dad is not in place \\
\hline 4 & Mother will beat Son 2 if Dad is not in place \\
\hline 5 & The criminals will hit Sons 1 if the Police are not in place \\
\hline 6 & Criminals will hit Sons 2 if Police are not in place. \\
\hline 7 & Criminals will beat Girl 1 if Police are not in place \\
\hline 8 & Criminals will beat Girl 2 if Police are not in place. \\
\hline 9 & The criminals will hit Dad if the Police are not in place \\
\hline 10 & Criminals will hit mother if the Police are not in place \\
\hline
\end{tabular}

Any state of the Crossing Rivers Problem could describe by (criminals, police, children, father and mother) and raft positions. The initial state and state of destination could describe as:

A. Initial state:

- Left area $=(1,1,1,1,1,1,1,1)$.

- Right area $=(0,0,0,0,0,0,0,0)$

- The raft position is on the left. That is, there are fathers, mothers, sons, daughters, police, and criminals in the left.

B. Goal state:

- $\quad$ Left area $=(0,0,0,0,0,0,0,0)$.

- Right area $=(1,1,1,1,1,1,1,1)$

- The position of the raft is on the right. That is, there are fathers, mothers, sons, daughters, police, and criminals in the right area

The process of development and search is extended by forming a new state from existing branches until the solution is achieved. The process of establishing a new state also performs checking procedures whether the situation has been previously set up in the tree. If it is already there, a new branch is not formed, and if it is not, then a new branch is formed. If there is no branch that can be developed again and it has not found a solution, then the problem being solved has no solution, the application of Breadth First Search algorithm on the problem of Crossing Rivers Problem is to find a solution how to move all the variables on the left to the right with Follow the predefined rule. From the variables and rules contained in the Crossing Rivers Problem, it can be concluded that some action can be done by keeping the existing regulations. The actions that can be done can be seen in Table 3 .

Table 3. Solution Crossing Rivers Problem

\begin{tabular}{|c|l|c|}
\hline Left Side & Action Solution & Right Side \\
\hline$(1,1,1,1,1,1,1,1)$ & - & $(0,0,0,0,0,0,0,0)$ \\
\hline$(1,1,1,1,1,1,0,0)$ & Police and Criminals cross to the right. & $(0,0,0,0,0,0,1,1)$ \\
\hline
\end{tabular}




\begin{tabular}{|l|l|l|}
\hline$(1,1,1,1,1,1,0,1)$ & The police crossed to the left. & $(0,0,0,0,0,0,1,0)$ \\
\hline$(0,1,1,1,1,1,0,0)$ & Police and Son 1 crossed to the right. & $(1,0,0,0,0,0,1,1)$ \\
\hline$(0,1,1,1,1,1,1,1)$ & Police and Criminals cross to the left. & $(1,0,0,0,0,0,0,0)$ \\
\hline$(0,0,1,1,0,1,1,1)$ & Father and Son 2 cross to the right. & $(1,1,0,0,1,0,0,0)$ \\
\hline$(0,0,1,1,1,1,1,1)$ & Dad crossed to the left. & $(1,1,0,0,0,0,0,0)$ \\
\hline$(0,0,1,1,0,0,1,1)$ & Mom and Dad crossed to the right. & $(1,1,0,0,1,1,0,0)$ \\
\hline$(0,0,1,1,0,1,1,1)$ & Mother crossed to the left. & $(1,1,0,0,1,0,0,0)$ \\
\hline$(0,0,1,1,0,1,0,0)$ & Police and Criminals cross to the right. & $(1,1,0,0,1,0,1,1)$ \\
\hline$(0,0,1,1,1,1,0,0)$ & Dad crossed to the left. & $(1,1,0,0,0,0,1,1)$ \\
\hline$(0,0,1,1,0,0,0,0)$ & Mom and Dad crossed to the right. & $(1,1,0,0,1,1,1,1)$ \\
\hline$(0,0,1,1,0,1,0,0)$ & Mother crossed to the left. & $(1,1,0,0,1,0,1,1)$ \\
\hline$(0,0,0,1,0,0,0,0)$ & Mother and Daughter 1 cross to right. & $(1,1,1,0,1,1,1,1)$ \\
\hline$(0,0,0,1,0,0,1,1)$ & Police and Criminals cross to the left. & $(1,1,1,0,1,1,0,0)$ \\
\hline$(0,0,0,0,0,0,1,0)$ & Police and Girl 2 cross to the right. & $(1,1,1,1,1,1,0,1)$ \\
\hline$(0,0,0,0,0,0,1,1)$ & The police crossed to the left. & $(1,1,1,1,1,1,0,0)$ \\
\hline$(0,0,0,0,0,0,0,0)$ & Police and Criminals cross to the right. & $(1,1,1,1,1,1,1,1)$ \\
\hline
\end{tabular}

The search for a solution in the tree tracing structure uses the search method of breadth-first search, so the solution (Table 3 ) obtained is the shortest and best option, some problem solving simulation Crossing River Problem made using programming language can be seen figure 2 and figure 3 .

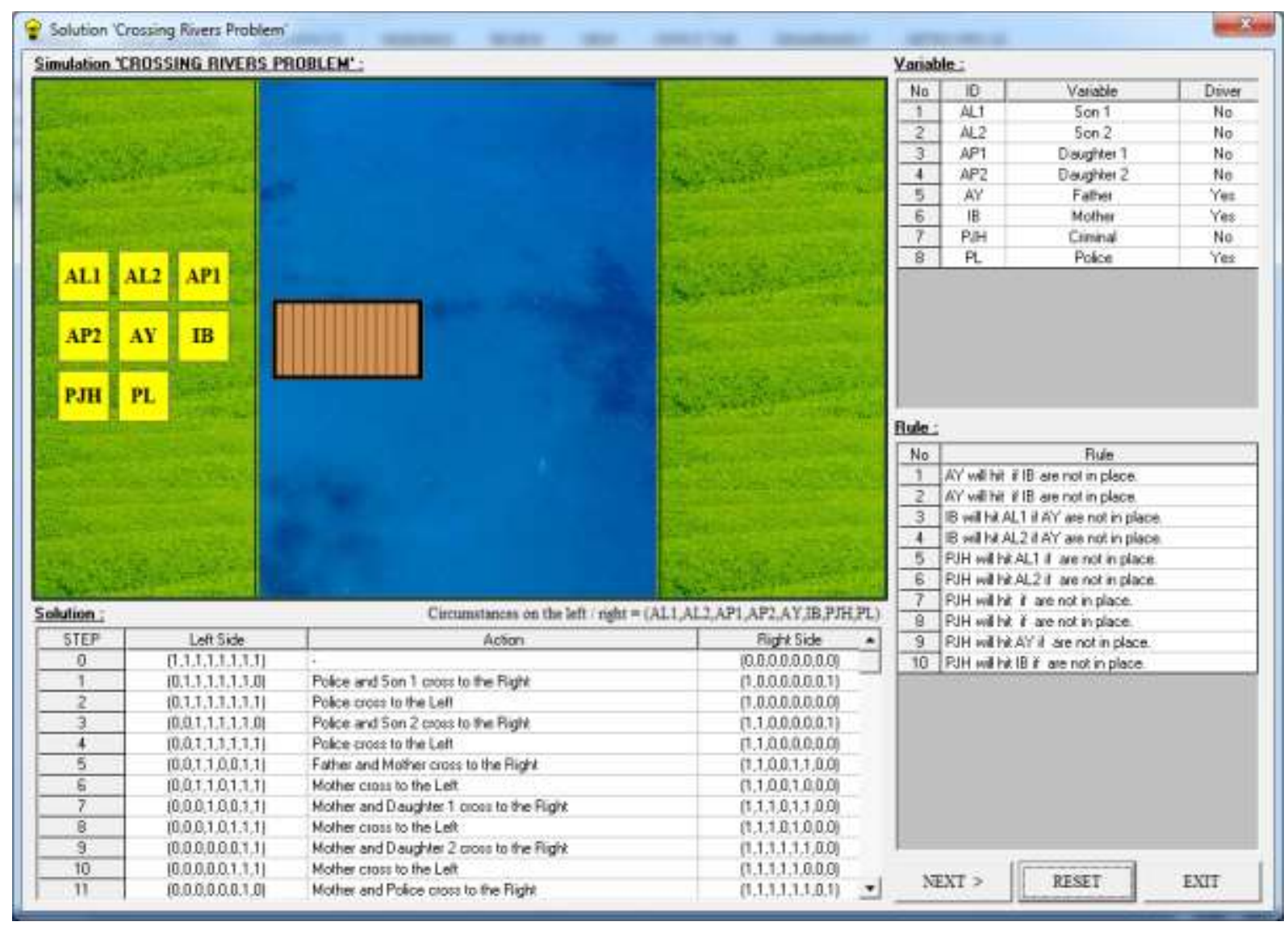

Figure 2. Initial State from Cross River Problem 
Figure 2 shows the initial condition of the simulation used to solve the problem of state and space crossing river problem with information of variable, rule and settlement process obtained from Breadth First Search algorithm.

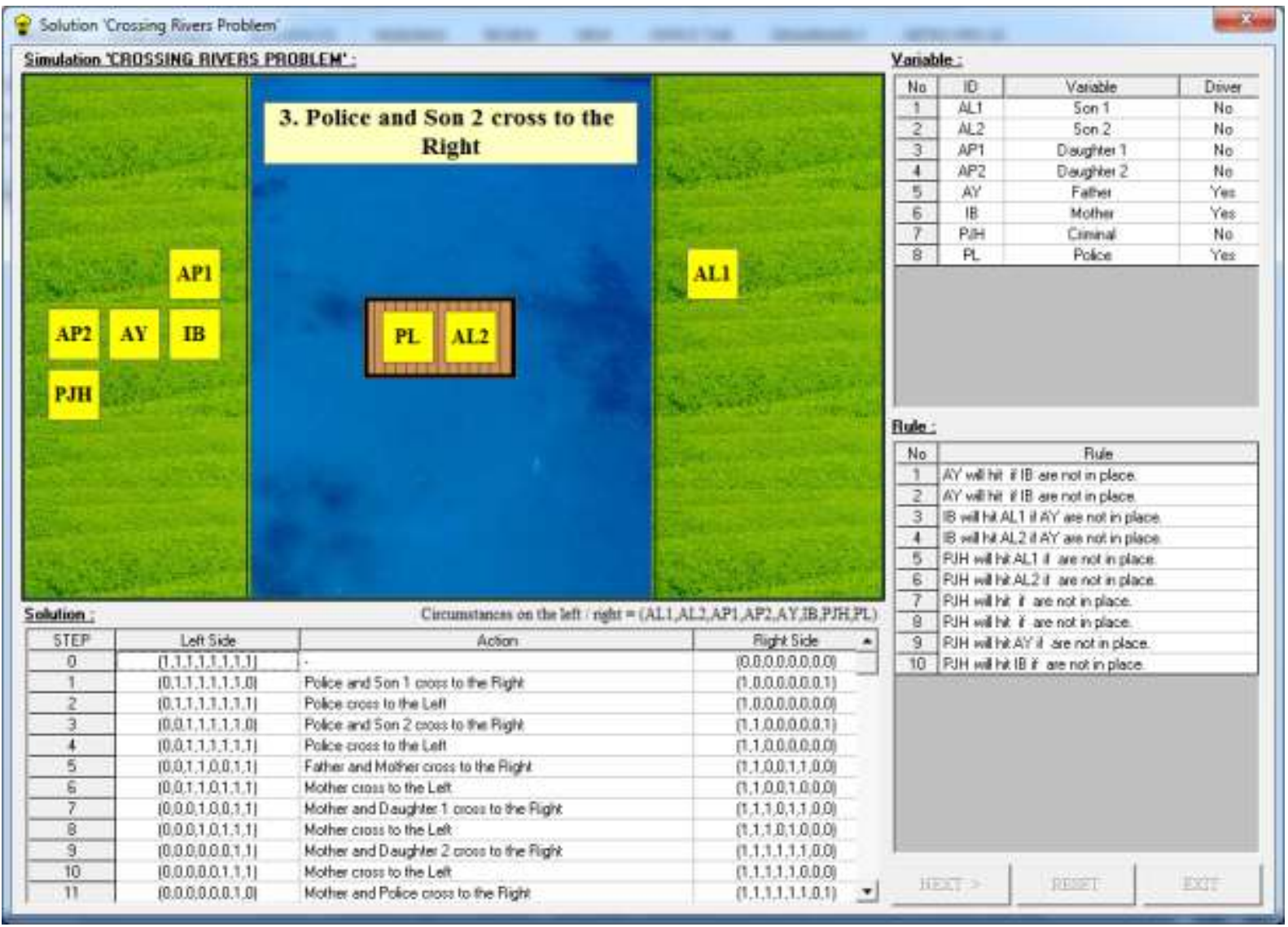

Figure 3. Simulation State and Space Cross River Problem

Figure 3 is a simulation process to solve the crossing river problem by performing the procedure accordance Breadth First Search algorithm.

\section{Conclusion}

After solving the problem of crossing rivers problem by using breadth-first search method can be concluded that a problem consists of variables and rules that can be utilized as an initial state to get the desired solution to achieve goal state, the problem used as an example in this paper could be used as a Preliminary solutions for various similar cases and application to computing systems will not be too difficult if the variables and rules already know.

\section{References}

[1] J. C. Trigeassou and N. Maamri, "State space modeling of fractional differential equations and the initial condition problem," in 6th International Multi-Conference on Systems, Signals and Devices, Djerba, Tunisia , 2009.

[2] B. Bonet and H. Geffner, "Planning as heuristic search," Journal Artificial Intelligence, vol. 129, no. $1-2$, pp. 5-33, 201. 
[3] S. Beamer, K. Asanović and D. Patterson, "Direction-Optimizing Breadth-First Search," Scientific Programming, vol. 21, no. 3-4, pp. 137-148, 2013.

[4] T. Kim and K. Chwa , "Parallel algorithms for a depth first search and a breadth first search," International Journal of Computer Mathematics, vol. 19, no. 1, pp. 39-54, 2007.

[5] A. Kaur, P. Sharma and A. Verma, "A appraisal paper on Breadth-first search, Depth-first search and Red black tree," International Journal of Scientific and Research Publications, vol. 4, no. 3, pp. 1-3, 2014.

[6] R. K. Ghosh and G. P. Bhattacharjee, "Parallel breadth-first search algorithms for trees and graphs," International Journal of Computer Mathematics, vol. 15, no. 1-4, pp. 255-268, 2007.

[7] R. Zhou and E. A. Hansen, "Breadth-first heuristic search," Journal Artificial Intelligence, vol. 170, no. 4-5, pp. 385-408, 2006. 